\title{
AUTOMATED EXTRACTION OF 3D TREES FROM MOBILE LIDAR POINT CLOUDS
}

\author{
Y. Yu ${ }^{\text {a }}$, J. Li $^{\text {b*, c }}$, H. Guan ${ }^{c}$, D. Zai ${ }^{a}$, C. Wang ${ }^{a}$ \\ ${ }^{a}$ Department of Computer Science, Xiamen University, 422 Siming Road South, Xiamen, FJ 361005, China - (allennessy.yu, \\ david102812)@gmail.com, cwang@xmu.edu.cn \\ ${ }^{\mathrm{b}}$ Key Laboratory of Underwater Acoustic Communication and Marine Information Technology (MOE), Xiamen University, 422 \\ Siming Road South, Xiamen, FJ 361005, China - junli@xmu.edu.cn \\ ${ }^{\mathrm{c}}$ Department of Geography and Environmental Management, University of Waterloo, 200 University Avenue West, Waterloo, ON \\ N2L 3G1, Canada - (h6guan, junli)@uwaterloo.ca
}

\section{Commission V, ICWG I/Va}

KEY WORDS: Mobile LiDAR, Tree Extraction, Shape Context, Segmentation, Point Cloud

\begin{abstract}
:
This paper presents an automated algorithm for extracting 3D trees directly from 3D mobile light detection and ranging (LiDAR) data. To reduce both computational and spatial complexities, ground points are first filtered out from a raw 3D point cloud via blockbased elevation filtering. Off-ground points are then grouped into clusters representing individual objects through Euclidean distance clustering and voxel-based normalized cut segmentation. Finally, a model-driven method is proposed to achieve the extraction of 3D trees based on a pairwise 3D shape descriptor. The proposed algorithm is tested using a set of mobile LiDAR point clouds acquired by a RIEGL VMX-450 system. The results demonstrate the feasibility and effectiveness of the proposed algorithm.
\end{abstract}

\section{INTRODUCTION}

Object detection and extraction from mobile light detection and ranging (LiDAR) point clouds has been a very active research topic in photogrammetry and remote sensing community. Most of existing methods are based on geometric features and reflectance properties of the objects to be handled. Yang et al. (2013b) proposed a principal component analysis (PCA) approach to separate trees and buildings from off-ground points. Then, building facades were fitted using the RANSAC algorithm. Finally, building facade footprints were extracted after projecting each fitted facade onto the horizontal plane, removing redundant segments, and connecting disjoint segments. Yu et al. (2014b) developed a pairwise 3D shape descriptor for modelling geometric features of 3D discrete points in manifold space. This $3 \mathrm{D}$ shape descriptor obtained promising results in extracting 3D light poles and trees from mobile LiDAR point clouds. Pu et al. (2011) proposed a percentile-based algorithm to extract pole-like objects, such as street light poles and traffic signposts, for road inventory mapping. Considering the attachments (e.g. advertising boards) and the shrubs, an off-ground object was first divided into quartiles. Then, the third quartile was selected and further partitioned into a set of slices. Finally, pole-like objects were recognized through the analysis of pole-like structures from the partitioned slices. Vertical profile analysis (Yang et al., 2013a; Guan et al., 2014) algorithms were developed to rapidly extract and delineate $3 \mathrm{D}$ urban roads from mobile LiDAR point clouds.

Rather than handling large-volume and highly dense point clouds in 3D space, some researchers projected and interpreted them into 2D geo-referenced feature images. Thus, object detection and extraction can be carried out based on 2D imagery using image processing methods. Considering elevation differences among street-scene objects, Yang et al. (2012) projected $3 \mathrm{D}$ point clouds into $2 \mathrm{D}$ geo-referenced elevation images through inverse distance weighted (IDW) interpretation. The generated feature images were used to extract street-scene objects, such as buildings and trees. Guan et al. (2014) rasterized the road surface points into a geo-referenced intensity image via an IDW interpretation method which utilized intensity information to interpret a group of points into a single pixel. The reflectance information in the generated images was used to extract road markings, which are highly retro-reflective objects painted on road surfaces, through intensity thresholding and morphological operations. Similarly, Yang et al. (2012) developed a model-based algorithm to extract rectangle-shaped road markings from the generated geo-referenced intensity images. $\mathrm{Yu}$ et al. (2014a) used marked point processes to extract road manhole and sewer well covers from georeferenced intensity images of road surfaces. Two types of marked points (disks and rectangles) and a set of transformations were defined to respectively model the geometric structures of road manhole and sewer well covers and their movements.

In this paper, we develop a novel algorithm to extract 3D trees directly from $3 \mathrm{D}$ mobile LiDAR point clouds. To reduce the quantity of the data to be handled, ground points are first removed from the raw point cloud using a block-based elevation filtering approach. Then, the isolated and unstructured offground points are further grouped and segmented into clusters representing individual objects through Euclidean distance clustering and voxel-based normalized cut segmentation, respectively. Finally, a model-driven method is developed to extract 3D trees of specific categories based on the similarity measurements between the prototype and each segmented object. The similarity between two point cloud objects is measured

\footnotetext{
* Corresponding author: junli@xmu.edu.cn.
} 
using a novel pairwise 3D shape descriptor, which can effectively and distinctively model the geometric structures of a 3D point within its vicinity. The proposed algorithm has been tested on a set of mobile LiDAR point clouds collected by a state-of-the-art RIEGL VMX-450 system. The experimental results obtained demonstrate the effectiveness and feasibility of the proposed algorithm in extracting 3D trees directly from mobile LiDAR point clouds.

\section{METHOD}

\subsection{Ground Removal}

Due to the design of mobile LiDAR systems, laser scanners usually have perfect views of the ground and roadside upward objects, such as buildings, trees, pole-like objects, etc. Thus, the collected point clouds contain a great portion of data points belonging to the ground. To reduce both computational and spatial complexities, it is necessary to filter out ground points before carrying out the extraction of 3D trees. In this paper, we propose a block-based elevation filtering approach to rapidly remove ground points.

Considering the perturbations of the ground in a large scene, a raw point cloud is first vertically divided into a group of blocks with a block size of $w$ b. These data blocks are processed separately to remove ground points. Such a strategy can effectively suppress the impacts of ground perturbations on the filtering results. Then, within each block, the point with the minimum elevation is ascertained and its elevation is selected as a height base for calculating the height of each data point. The height of a point is defined as the elevation difference between this point and the point with the minimum elevation within the same block. Finally, the points with heights smaller than a predefined threshold $h_{\mathrm{g}}$ are regarded as ground points, and further removed. After processing all blocks, the ground points are basically filtered out. Figure 1 shows a visual example of the off-ground points obtained using the proposed block-based elevation filtering approach.

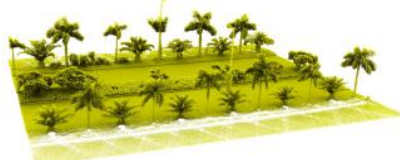

(a)

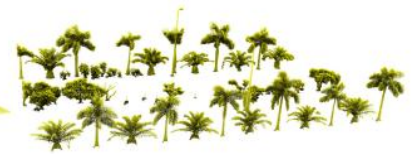

(b)
Figure 1. (a) Raw point cloud, and (b) off-ground points.

\subsection{Off-Ground Point Clustering and Segmentation}

The off-ground points obtained through ground removal are still isolated and unorganized. Therefore, the points belonging to a specific object need to be further grouped before the identification of $3 \mathrm{D}$ trees. In this paper, we first adopt a Euclidean distance clustering approach to group the discrete off-ground points into clusters. The Euclidean distance clustering approach utilizes the Euclidean distances between each pair of points to group them into a set of clusters based on a clustering distance $d_{c}$. Particularly, an un-clustered point is contained into a specific cluster if and only if its shortest Euclidean distance to the points within this cluster lies below $d_{\mathrm{c}}$. Otherwise, a new clustering operation will start at this point. Such a clustering approach can rapidly provide an initial and promising clustering result for the off-ground points. To further reduce the searching regions, the small clusters unlikely to be trees are eliminated. This is achieved by calculating a bounding box for each cluster and eliminating those clusters with a diagonal of their bounding boxes smaller than a pre-defined threshold. Figure 2(a) presents the clustering result after eliminating small segments. Different colours represent different clusters.

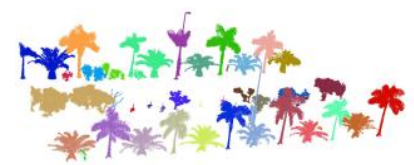

(a)

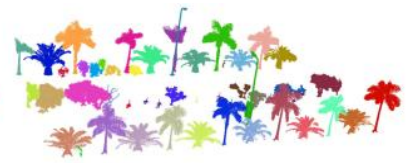

(b)
Figure 2. (a) Off-ground points clustering result, and (b) segmentation result of adjacent or overlapped objects.

However, as shown in Figure 2(a), some clusters contain multiple objects. This is because the Euclidean distance clustering approach cannot separate the adjacent or overlapped objects based on only distance measurements. To further segment such clusters, we introduce a voxel-based normalized cut segmentation method (Shi and Malik, 2000). First, the clusters containing more than one objects are partitioned into an octree structure with a spacing of $w_{\mathrm{o}}$. Then, the partitioned voxels are grouped into a weighted graph $\boldsymbol{G}=\{\boldsymbol{V}, \boldsymbol{E}\}$, where nodes $\boldsymbol{V}$ are constructed by the voxels and edges $\boldsymbol{E}$ are linked between each pair of nodes. The weight $w_{i j}$ on the edge connecting nodes $i$ and $j$ is defined to measure the similarity between these two nodes. It is defined as follows:

$$
w_{i j}=\left\{\begin{array}{c}
\exp \left(-\frac{\left\|p_{i}^{\mathrm{H}}-p_{j}^{\mathrm{H}}\right\|_{2}^{2}}{\sigma_{\mathrm{H}}^{2}}\right) \cdot \exp \left(-\frac{\left|p_{i}^{\mathrm{V}}-p_{j}^{\mathrm{V}}\right|^{2}}{\sigma_{\mathrm{V}}^{2}}\right), \text { if }\left\|p_{i}^{\mathrm{H}}-p_{j}^{\mathrm{H}}\right\|_{2} \leq d_{\mathrm{H}},(1) \\
0, \text { otherwise }
\end{array}\right.
$$

where $p_{i}=\left(x_{i}, y_{i}, z_{i}\right)$ and $p_{j}=\left(x_{j}, y_{j}, z_{j}\right)$ denote the centroids of voxels $i$ and $j$, respectively; $p_{\mathrm{i}}^{\mathrm{H}}=\left(x_{i}, y_{i}\right)$ and $p_{j}^{\mathrm{H}}=\left(x_{j}, y_{j}\right)$ are the projections of the centroids on the horizontal plane; $p_{i}^{\mathrm{V}}=z_{i}$ and $p_{j}^{\mathrm{V}}=z_{j}$ are the $z$ coordinates of the centroids; $\sigma_{\mathrm{H}}$ and $\sigma_{\mathrm{V}}$ are the standard deviations of the horizontal and vertical distributions, respectively; $d_{\mathrm{H}}$ is a distance threshold for determining the maximum horizontal distance between two voxels.

Then, the computed weights of graph $\boldsymbol{G}$ are used to construct a generalized eigenvalue problem (Shi and Malik, 2000) for segmenting the voxels into disjoint groups representing individual objects:

$$
(\mathbf{D}-\mathbf{W}) y=\lambda \mathbf{D} y,
$$

where $\mathbf{W}_{i j}=w_{i j}$, and $\mathbf{D}$ is a diagonal matrix with $\mathrm{D}_{i i}=\Sigma_{m} w_{i m}$.

Finally, by applying a threshold to the eigenvector associated with the second smallest eigenvalue, the clusters are segmented into two segments. Figure 2(b) shows the segmented clusters using the proposed voxel-based normalized cut segmentation method.

\subsection{Tree Extraction}

We develop a model-driven method to extract trees of specific categories from the segmented off-ground objects. As shown in Figure 3(a), a tree model is selected as a template for matching the objects having similar features with the model. To this end, the model and each of the segmented off-ground objects are uniformly sampled a set of feature points. In order to compute a 
one-to-one matching between the feature points on the model and an off-ground object, the numbers of feature points are set to be the same for the model and the off-ground object, respectively. Then, each feature point is described using the pairwise 3D shape context proposed by $\mathrm{Yu}$ et al. (2014b). Denote $H_{p}=\left[h_{1}^{p}, h_{2}^{p}, \ldots h_{N-1}^{p}\right] \in R^{K_{b} \times(N-1)}$ as the pairwise 3D shape context for feature point $p$. $N$ denotes the number of feature points, and $K_{\mathrm{b}}$ denotes the number of bins for constructing the pairwise 3D shape context (Yu et al., 2014b). The matching cost between a pair of feature points $p$ and $q$ is defined as:

$$
C(p, q)=\min _{i, j=1}^{N-1} \frac{1}{2} \sum_{k=1}^{K_{\mathrm{b}}} \frac{\left[h_{i}^{p}(k)-h_{j}^{q}(k)\right]^{2}}{h_{i}^{p}(k)+h_{j}^{q}(k)} .
$$

The dissimilarity between the model $\mathrm{P}$ and an off-ground object $\mathrm{Q}$ is defined as:

$$
\operatorname{DIS}(P, Q)=M_{P, Q}(\pi)+G(P, Q),
$$

where $\mathrm{M}_{\mathrm{P}, \mathrm{Q}}(\pi)$ computes the one-to-one matching costs between the feature points on the model $\mathrm{P}$ and the feature points on the off-ground object Q; $\mathrm{G}(\mathrm{P}, \mathrm{Q})$ measures the global dissimilarity between $\mathrm{P}$ and $\mathrm{Q}$. They are defined as follows:

$$
\begin{gathered}
M_{P, Q}(\pi)=\min _{\pi} \frac{1}{N} \sum_{i=1}^{N} C(i, \pi(i)), \\
G(P, Q)=\frac{1}{N(N-1)} \sum_{i=1}^{N} \sum_{j=1}^{N-1} \min _{m, n}\left\|H_{p_{i}}(j)-H_{q_{m}}(n)\right\|_{1} .
\end{gathered}
$$

Finally, the dissimilarity measurements from all off-ground objects are thresholded to achieve the extraction of the trees. Figure 3(b) presents the extracted 3D trees using the model in Figure 3(a).

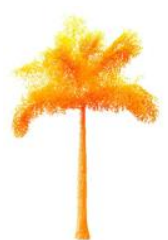

(a)

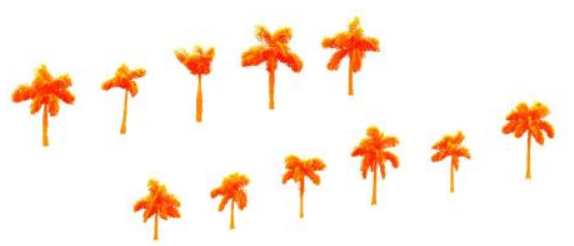

(b)
Figure 3. (a) Tree model, and (b) extracted 3D trees.

\section{RESULTS AND DISCUSSION}

\subsection{System}

The mobile LiDAR point cloud data used in this study were collected using a state-of-the-art RIEGL VMX-450 mobile LiDAR system. As shown in Figure 4, this system is smoothly integrated with two full-view RIEGL VQ-450 laser scanners, four high-resolution $\mathrm{CCD}$ cameras, two global navigation satellite systems (GNSSs), an inertial measure unit (IMU), and a wheel mounted distance measurement indicator (DMI). The two laser scanners are configured with an " $\mathrm{X}$ " pattern for providing a better coverage of the surveyed scenes. The scanners rotate to emit laser beams with a maximal measurement rate of about 1.1 million measurements per second, a line scan speed of up to 400 scans per second, and a maximal valid range of approximately 800 meters. The accuracy and precision of the acquired point clouds are within $8 \mathrm{~mm}$ and $5 \mathrm{~mm}$, respectively.
The mobile LiDAR point cloud data were collected on Ring Road South in City of Xiamen, China. It is a typical tropical urban area with high-rise buildings, dense vegetation, and various traffic utilities. The entire mapping system was mounted on the roof of a minivan (see Figure 4). The driving speed was limited within $50 \mathrm{~km} / \mathrm{h}$. With such a mapping speed, the point density close to the scanner centre is about 7000 points $/ \mathrm{m}^{2}$. The highly dense and accurate point cloud data are a promising data source for extracting $3 \mathrm{D}$ trees.

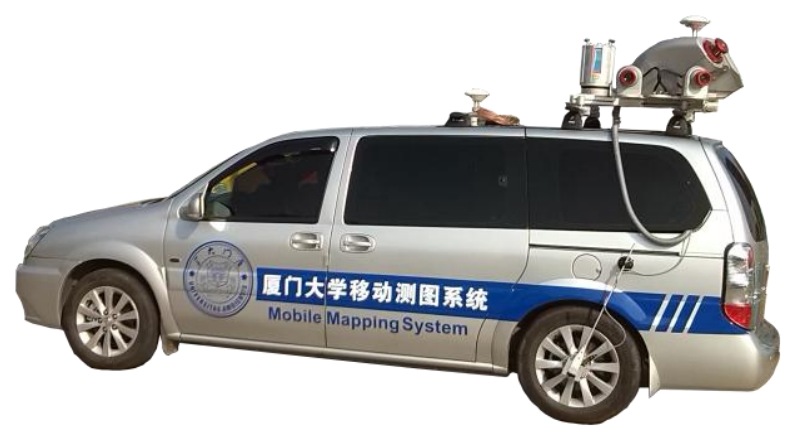

Figure 4. RIEGL VMX-450 mobile LiDAR system.

\subsection{D Tree Extraction}

Three datasets containing different categories of trees were selected for the evaluation of the proposed algorithm, as shown in Figures 5(a), 6(a), and 7(a), respectively. First, ground points were removed from the point clouds using the block-based filtering method with a block size $w_{\mathrm{b}}=3 \mathrm{~m}$ and a height threshold $h_{\mathrm{g}}=0.4 \mathrm{~m}$. Then, the off-ground points were further clustered via the Euclidean distance clustering approach with a distance $d_{\mathrm{c}}=0.15 \mathrm{~m}$ and segmented into individual objects based on the voxel-based normalized cut segmentation method with parameter configurations of $w_{\mathrm{o}}=0.1 \mathrm{~m}, \sigma_{\mathrm{H}}=2 \mathrm{~m}, \sigma_{\mathrm{V}}=10$ $\mathrm{m}$, and $d_{\mathrm{H}}=5 \mathrm{~m}$. Next, each model and each off-ground object were uniformly sampled $N=20$ points which were modelled using the pairwise 3D shape context. Finally, 3D trees were extracted from the segmented off-ground points based on the dissimilarity measurements between the model and the offground objects. The extracted $3 \mathrm{D}$ trees in each dataset were respectively presented in Figures 5(b), 6(b), and 7(b). The extraction results demonstrate that the proposed algorithm achieves promising performance in extracting $3 \mathrm{D}$ trees directly from 3D mobile LiDAR point clouds.

\section{CONCLUSION}

In this paper, we have proposed a novel algorithm for extracting $3 \mathrm{D}$ trees directly from $3 \mathrm{D}$ mobile LiDAR point clouds. We developed a block-based ground removal method to rapidly filter out ground points. To group the isolated and unstructured off-ground points into specific object representations, we combined Euclidean distance clustering and voxel-based normalized cut segmentation to organize discrete points into clusters and segment the clusters containing multiple objects into individual objects. Finally, a 3D object extraction method was developed for extracting 3D trees from the segmented offground objects based on a pairwise 3D shape context. The proposed algorithm has been tested on three mobile LiDAR point cloud datasets with different categories of trees. The extraction results have demonstrated the feasibility and effectiveness of the proposed algorithm in extracting 3D trees directly from $3 \mathrm{D}$ point clouds. 


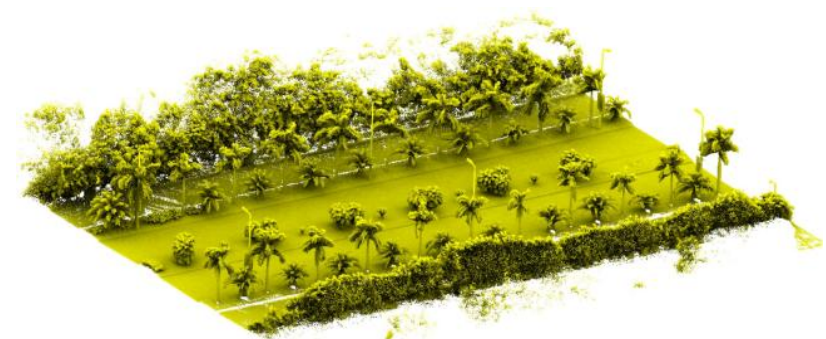

(a)

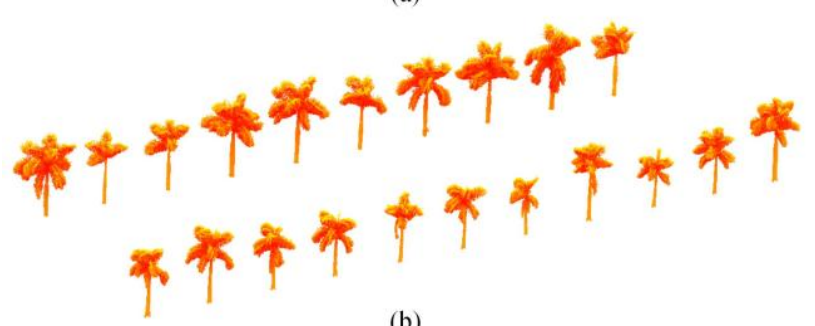

(b)

Figure 5. (a) Dataset I, and (b) extracted 3D trees.

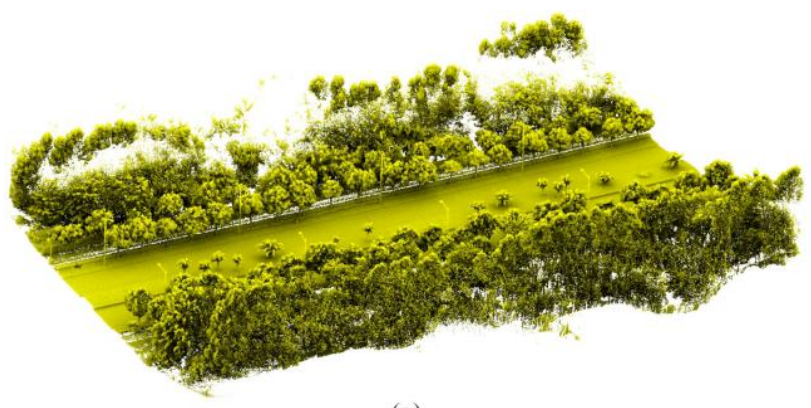

(a)

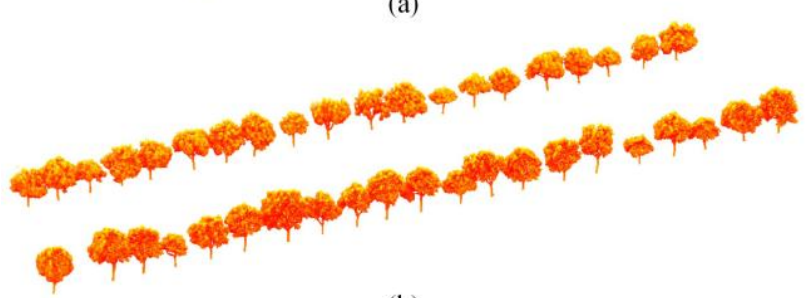

(b)

Figure 6. (a) Dataset II, and (b) extracted 3D trees.

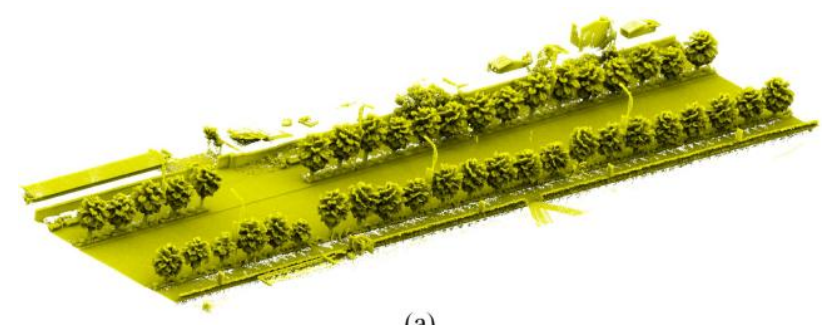

(a)

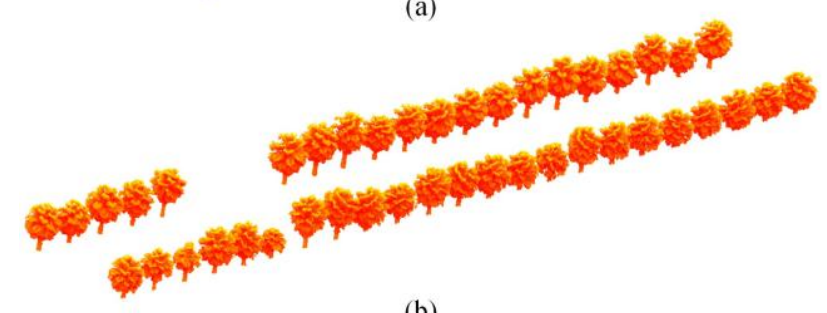

(b)

\section{REFERENCES}

Guan, H., Li, J., Yu, Y., Wang, C., Chapman, M., Yang, B., 2014. Using mobile laser scanning data for automated extraction of road markings. ISPRS Journal of Photogrammetry and Remote Sensing, 87, pp. 93-107.

Pu, S., Rutzinger, M., Vosselman, G., Elberink, S.O., 2011. Recognizing basic structures from mobile laser scanning data for road inventory studies. ISPRS Journal of Photogrammetry and Remote Sensing, 66, pp. S28-S39.

Shi, J., Malik, J., 2000. Normalized cuts and image segmentation. IEEE Transactions on Pattern Analysis and Machine Intelligence, 22(8), pp. 88-905.

Williams, K., Olsen, M.J., Roe, G.V., Glennie, C., 2013. Synthesis of transportation applications of mobile LiDAR. Remote Sensing, 5(9), pp. 4652-4692.

Yang, B., Fang, L., Li, Q., Li, J., 2012. Automated extraction of road markings from mobile LiDAR point clouds. Photogrammetric Engineering and Remote Sensing, 78(4), pp. 331-338.

Yang, B., Fang, L., Li, J., 2013a. Semi-automated extraction and delineation of 3D roads of street scene from mobile laser scanning point clouds. ISPRS Journal of Photogrammetry and Remote Sensing, 79, pp. 80-93.

Yang, B., Wei, Z., Li, Q., Li, J., 2012. Automated extraction of street-scene objects from mobile LiDAR point clouds. International Journal of Remote Sensing, 33(8), pp. 5839-5861.

Yang, B., Wei, Z., Li, Q., Li, J., 2013b. Semiautomated building facade footprint extraction from mobile LiDAR point clouds. IEEE Geoscience and Remote Sensing Letters, 10(4), pp. 766-770.

Yu, Y., Li, J., Guan, H., Wang, C., Yu, J., 2014a. Automated detection of road manhole and sewer well covers from mobile LiDAR point clouds. IEEE Geoscience and Remote Sensing Letters, 11(9), pp. 1549-1553.

Yu, Y., Li, J., Yu, J., Guan, H., Wang, C., 2014b. Pairwise three-dimensional shape context for partial object matching and retrieval on mobile laser scanning data. IEEE Geoscience and Remote Sensing Letters, 11(5), pp. 1019-1023.

Figure 7. (a) Dataset III, and (b) extracted 3D trees. 